\title{
Oftalmologia e realidade virtual
}

\author{
Virtual Reality and ophthalmology
}

Jânio Araruna Carvalho'

\section{$\underline{\text { RESUMO }}$}

Objetivo: Identificar como os simuladores estão sendo usados para ensino, treinamento, terapia e exames na medicina, com destaque para oftalmologia. A realidade virtual surgiu como uma ferramenta auxiliar no ensino e treinamento médico. Na oftalmologia, o progresso dessa técnica tem sido publicado em algumas revistas de saúde. Baseado nessas fontes, investigamos como a Oftalmologia tem interagido com a Realidade Virtual, essa ferramenta que veio para auxiliar o ensino da Medicina. Métodos: Revisão sistemática de artigos das principais revistas, além de livros sobre o assunto entre 1995 e 2010. Revisamos a literatura publicada dos últimos quinze anos baseados em referências mencionadas em várias revistas, como exemplo podemos citar a Medical Education, Journal of the American College of Surgeon, Computers \& Graphics, New England Journal of Medicine, Proceedingof the IEEE no período de 1990 a 2010. Resultados: Após a revisão de 200 artigos encontrados sobre o tema educação médica e realidade virtual publicados em revistas nacionais e internacionais entre os anos de 1995 e 2010, percebemos as diferenças entre as áreas da medicina, no que se refere ao uso da realidade virtual (RV). Apenas 10 artigos explicitam com algum aprofundamento os simuladores oftalmológicos. Conclusão: A oftalmologia é uma das áreas que menos interagem com a realidade virtual, devido a dificuldades de montagem de imagens para serem aplicadas no simulador. Mas é uma das áreas que mais precisa, devido à delicadeza da estrutura de trabalho, olho humano. Assim, a Realidade Virtual (RV) tem aparecido na otoscopia virtual e na formação dos atlas virtuais. Na cirurgia, os simuladores ainda são poucos, mas provavelmente será a área mais promissora, devido as vantagens do treinamento virtual. Assim, a RV tem surgido junto aos serious games, ensino e treinamento de oftalmologia, além de terapia de fobias.

Descritores: Simulação por computador; Educação médica/métodos; Modelos educacionais; Interface usuário, computador; Educação baseada em competências

\section{Abstract}

Objective: To identify how the simulators are being used for education, training, therapy and tests in medicine, particularly ophthalmology. Virtual reality has emerged as an auxiliary tool in education and medical training. In ophthalmology, the progress of this technique has been published in some health magazines. Based on these sources, we investigated the ophthalmology have interacted with virtual reality, this tool that came to support the teaching of medicine. Methods: A systematic review of articles in leading journals on the subject between 1995 and 2010 as well as books on the subject. Results: After reviewing 200 articles on medical education and virtual reality published in national and international journals between 1995 and 2010 we see the differences between the areas of medicine with regard to the use of VR. Only 10 articles with explicit deepening ophthalmic simulators. Conclusion: Ophthalmology is one of the areas least interact with virtual reality, due to difficulties of assembling images for application in the simulator. But it is one of the areas most in need, because the delicacy of the work structure, the human eye. Thus, the Virtual Reality (VR) has asparecido in virtual otoscopy and formation of virtual atals. At surgery, the simulators are still few, but will probably be the most promising area because of the advantages of virtual training. Thus, RV has appeared next to serious games, education and training of ophthalmology, and treatment of phobias.

Keywords: Computer, simulation; Education, medical/methods; Models, educational; User-computer interface; Competency-based education/methods

${ }^{1}$ Universidade Federal da Paraíba - UFPB - João Pessoa (PB), Brasil.

Os autores declaram inexistir conflitos de interesses

Recebido para publicação em: 7/6/2010 - Aceito para publicação em 6/12/2011 


\section{INTRODUÇÃO}

$\Delta$ Realidade Virtual é uma ferramenta que usa a simulação de uma situação real e a transforma em uma igual situação que pode ser feita virtualmente, imitada através das tecnologias da informática.

O termo Realidade Virtual refere-se a uma experiência imersiva e interativa baseada em imagens gráficas tridimensionais geradas em tempo real por computador. O principal objetivo desta nova tecnologia é fazer com que o participante desfrute de uma sensação de presença no mundo virtual. Para propiciar esta sensação de presença o sistema de RV integra sofisticados dispositivos. Estes dispositivos podem ser luvas de dados, óculos, capacetes, etc ${ }^{(1)}$

Este trabalho buscou saber como diversas áreas médicas tem utilizado a Realidade Virtual (RV) em seu dia a dia. Seja usando essa ferramenta para o ensino da medicina, o treinamento dos médicos, a terapia e reabilitação dos pacientes e chamado exame virtual. Revisamos a literatura publicada dos últimos quinze anos baseado em referências de várias revistas, como exemplo: Institute of Electrical and Electronics Engineers (IEEE), The New England Journal of Medicine, Medical Education e American Journal of Obstetrics and Gynecology de 1990 a 2010 e pesquisa em livros como Medicine Meets Virtual Reality (MMVR), teses de mestrado_resultando em 200 artigos lidos integralmente.

O simulador seria uma ferramenta que busca imitar uma situação real, com a finalidade de facilitar o treinamento e a aprendizagem. Existem os simuladores que usam apenas o computador (programas de computador) e os que usam acessórios, como os hápticos e outros hardwares.

No texto buscamos classificar simuladores em quatro grupos: ensino, treinamento e planejamento, terapia e exame.

- Ensino: pode ser "apenas" uma visualização de estruturas anatômicas, por exemplo;

- Treinamento: simuladores mecânicos e computacionais, por exemplo.;

- Planejamento: planejamento de uma cirurgia ou de radioterapia;

- Terapia: reabilitação, como os serious games e tratamento de fobias;

- Exame: imagens obtidas exclusivamente para um paciente, como colposcopia virtual, por exemplo.

Já afirmava que a realidade virtual seria uma das formas de ensinar medicina. Isso vem sendo confirmado por diversos autores ${ }^{(2)}$. Afirma-se que todas as áreas da medicina vão se beneficiar com o ensino com imagens $3 \mathrm{D}$, simuladores e robôs. Isso permitirá uma medicina mais eficiente, médicos mais treinados e deixando de lado até o uso de manequins, além de poupar o paciente das mãos inexperientes dos alunos e diminuir o uso dos cadáveres para aulas de medicina ${ }^{(3)}$.

Mais recentemente ${ }^{(4)}$, afirma que o uso da simulação virtual tem sido proposto como o próximo grande passo na evolução educação na saúde , mas precisamos vencer as barreiras, as quais citamos algumas neste texto.

A maior divulgação da ocorrência de erros médicos e das limitações do ensino convencional têm gerado forte pressão da sociedade para melhoria da educação médica. Desenvolveram-se novos conceitos éticos na sociedade, e o "aprender fazendo" não é mais tolerado na maioria dos países desenvolvidos. Em alguns países até mesmo o treinamento em animais foi banido. Em 2004, a Sociedade Americana de Emergência expressamente recomendou que a simulação fosse implementada em diversas atividades envolvendo o treinamento de médicos residentes. Isso mostra o potencial da medicina juntamente com a computação sendo reconhecido ${ }^{(3)}$.

A simulação avançou muito também com uso de robôs.A cirurgia robótica assistida é uma experiência onde o médico a quilômetros de distância envia comandos e o robô opera, permitindo que o cirurgião possa realizar o procedimento minimamente invasivo, com maior precisão e com potenciais benefícios para os pacientes ${ }^{(5)}$.

Será uma medicina muito útil principalmente para neurocirurgia, devido à precisão necessária nessa especialidade da Medicina.

O número de artigos sobre o assunto vem aumentando a cada ano desde 1995. Em 1998 já declarava que com hápticos inovadores, como dispositivos de interface e treinamento baseado em computador iria permitir que tanto os médicos inexperientes para a prática de novos procedimentos como os médicos com experiência pudessem aprender técnicas modernas ${ }^{(6)}$.

Soferman (1998) já percebia que a medicina virtual exigia uma tecnologia sofisticada e uma colaboração entre profissionais de diferentes áreas, sabendo-se que a medicina é complexa e a tecnologia é limitada ${ }^{(7)}$.

Realidade virtual tem aplicação em qualquer circunstância em que ensaios repetidos são necessários para a aquisição de habilidades e as circunstâncias clínicas de ensino são infrequentes ${ }^{(8)}$.

Em 1998 já existiam alguns softwares usados no pós-operatório, na reabilitação e na psiquiatria com bons resultados $^{(2)}$. O progresso continua, Nunes et al. ampliam o seu uso para promoção de saúde e condicionamento físico. Cita também o uso em tratamentos psicológicos, 
acompanhamento de fobias, medo de altura ou de insetos. Estas aplicações tentam reproduzir situações de forma a habituar o usuário por meio da experimentação ${ }^{(9)}$.

A realidade virtual pode ser usada de forma divertida. Savi et al. cita seu uso no entretenimento, com jogos que educam e chamam atenção muito mais que uma aula expositiva. Eles ensinam conteúdos das disciplinas e promovem o desenvolvimento de habilidades importantes para ampliar a capacidade cognitiva e intelectual ${ }^{(10)}$.

Neste mundo "simulado" os usuários podem mover e interagir como se estivessem no mundo real ${ }^{(11)}$.

A capacidade de criar clínicas ou situações através de simulações de realidade virtual podem facilitar o cumprimento das metas de um treinamento tradicional ${ }^{(8)}$.

Temos ainda os simuladores de exames. Os exames virtuais da medicina foram criados para dar um suporte a mais na procura por lesões. Juntando as imagens da TC, forma-se uma imagem 3D. São usados com a finalidade de orientação específica para aquele paciente que vai se submeter a um procedimento.

Nosso texto busca se interar em que áreas a realidade virtual vem se aprofundando e como ela vem se tornando muito mais que uma simples ferramenta auxiliar da medicina. A Realidade Virtual não pode ser tratada apenas como "mais uma ferramenta" para melhorar a aprendizagem e sim, como um poderoso instrumento de aprendizagem onde os métodos tradicionais estão falhando ${ }^{(12)}$. Em algumas áreas a RV tem se sobreposto, mas não em todas.

\section{MÉTOdos}

Revisão sistemática de artigos das principais revistas sobre o assunto entre 1995 e 2010, além de livros sobre o assunto. Revisão bibliográfica nos 200 artigos encontrados nas fontes: revistas Medical Education, Journal of the American College of Surgeon, Computers \& Graphics, New England Journal of Medicine, Proceedingos of the IEEE. A busca foi feita por meio dos descritores Virtual reality e Ophthalmology.

\section{Resultados}

Depois de revermos 200 artigos sobre educação médica e realidade virtual publicados em revistas nacionais e internacionais entre os anos de 1990 e 2010, percebemos as diferenças entre as áreas da medicina no que se refere ao uso da RV. Uma minoria de artigos explicitam com aprofundamento os simuladores oftalmológicos. A maioria não cita simuladores que en- volvem a oftalmologia e portanto não são usados para treinamento na área.

\section{DısCUSSÃO}

Para que simular?

A simulação é uma técnica de ensino que se fundamenta em princípios do ensino baseado em tarefas (EBT) (em inglês, SBME) e se utiliza da reprodução parcial ou total destas tarefas em um modelo artificial, conceituado como simulador. Sua aplicação é relacionada, em geral, a atividades práticas,que envolvam habilidades manuais ou decisões. Historicamente se desenvolveu isoladamente em diversas áreas do conhecimento humano e apenas recentemente começa a ser sistematizada ${ }^{(3)}$. É importante lembrar que o conteúdo educacional é adaptado à tecnologia. Quanto maior a potência do computador maior será o nível de realismo ${ }^{(2)}$.

$\mathrm{O}$ advento da computação de alto poder real e as representações gráficas do tempo permite a medicina avançar além do método tradicional de ensino e de começar a educar médicos sem colocar pacientes em risco(2).

Algumas faculdades já não usam mais cadáveres no ensino da anatomia humana e usam muitos simuladores para poupar o treinamento desnecessário em pacientes. Mas os cadáveres não vão sumir da medicina tão rápido, pois existe a limitação gráfica e algumas estruturas são melhores estudadas na peça humana real.

Nas categorias de treinamento, exame e ensino quem usa o simulador pode ser um médico, um enfermeiro, ou outro profissional ${ }^{(11)}$. Na terapia o usuário é o paciente em reabilitação.

No ensino e treinamento o uso de técnicas de simulação permitem ao estudante adquirir habilidades variadas, repetindo os procedimentos diagnósticos ou terapêuticos, tantas vezes quanto forem necessárias até atingir o estágio de domínio. A execução da tarefa pode e deve ser observada, de modo que as devidas correções sejam feitas de imediato e o estudante receba os devidos comentários ("feedback") sobre a sua atuação.

O uso de simuladores na educação tem como objetivo popularizar as oportunidades. A partir do simulador, muitos médicos podem aprender um procedimento específico, algo que não é possível hoje. Se um paciente chega para a cirurgia de vesícula biliar, por exemplo, apenas os residentes que trabalham com o médico do corpo docente podem aprender o procedimento. Com o aprendizado incorporado em um simulador, muitos estudantes podem participar da operação, das complicações e da partilha de informações ${ }^{(6)}$. 


\section{Estereoscopia}

O termo estereoscopia é bastante usado em simulação virtual.

A profundidade, gerada pela visão binocular é dada pela diferença de ângulos com que as imagens são percebidas. Ao apresentar aos olhos duas imagens de um mesmo objeto, de pontos de vista diferentes, e conseguir por algum artifício, fazer com que cada olho capte somente a imagem colocada à sua frente, o cérebro, ao receber as duas imagens distintas, interpreta-as como as imagens que receberia se observado o objeto diretamente, e as funde em uma única imagem tridimensional, realizando a visão binocular natural.

A estereoscopia pode ser entendida como a fusão, feita no cérebro, de duas imagens bidimensionais resultantes da projeção planar de uma cena tridimensional . Então, para a criação de uma cena estereoscópica é necessário um conjunto de técnicas que permitirão gerar um par de imagens,denominado par estéreo, da mesma cena. Em seguida, é necessário o uso de equipamentos especiais para a visualização individual de cada imagem deste par para cada um dos olhos. Alguns dos principais dispositivos para visualização estereoscópica são os óculos com filtros coloridos, óculos com filtros polarizados, os "head mounted displays" (HMD) e os óculos obturadores (shutter glasses).

\section{Serious games: treinamento, ensino e terapia.}

Os jogos sérios como são conhecidos têm sido usados em várias áreas, incluindo a oftalmologia. Pacientes com deficiência visual podem treinar habilidades brincando com esses jogos.

As instituições de ensino estão ampliando o uso das tecnologias de informação e comunicação para oferecer aos alunos mídias interativas que possam enriquecer as aulas. Os jogos digitais aparecem nesse contexto como um recurso didático que contém características que podem trazer uma série de benefícios para as práticas de ensino e aprendizagem ${ }^{(10)}$.

Os serious games são classes de jogos que visam treinar profissionais, conscientização e simular situações críticas em geral. Resgata-se a realidade virtual e aproxima-a da sociedade. Existem em várias plataformas como um jogo eletrônico, como computadores, consoles (videogames), miniconsoles (hand-helds), e dispositivos móveis (celulares, palms) que podem ser usados em medicina para, por exemplo, aumentar a percepção de pessoas que sofreram AVC (acidente vascular cerebral) ${ }^{(9)}$.

Segundo Savi et al. os serious games têm efeito motivador, facilitador do aprendizado, desenvolvimento de habilidades cognitivas, aprendizado por descober- ta, experiência de novas identidades, socialização, coordenação motora e comportamento expert. E usam tecnologias mais simples. Por isso são muito bem aceitos na Medicina, com muitas utilidades ${ }^{(10)}$.

Como exemplo tem o Re-Mission11, um jogo para jovens com câncer que procura ser tanto divertido como informativo e, em vinte níveis, esclarece para os pacientes os diferentes tipos de tratamento, como eles funcionam, e a importância de realizá-los. O jogador controla um nano-robô que é injetado dentro dos corpos de pacientes e luta contra as infecções celulares do câncer. Além disso, deve monitorar a saúde do paciente virtual e informar seus sintomas para a médica dentro do jogo ${ }^{(10)}$.

\section{Simuladores}

O simulador começou a ser usado pelo pessoal de defesa militar, aeronáutica e aviação e foi ganhando espaço na saúde de uma forma bem flexível.

Diversos estímulos têm fomentado o desenvolvimento da simulação na área médica ${ }^{(3)}$. Por isso vários sistemas educacionais têm sido propostos. Os quais utilizam tecnologias de computação, incluindo técnicas multimídia e uso de ambientes virtuais imersivos ${ }^{(13)}$.

\section{Terapias}

O sistema de realidade aumentada para educação de portadores de necessidades especiais é um dos usos da RV.

\section{Terapia: Simulador de altura}

Mergulhão cita para o uso de RV em hospitais nos Estados Unidos como ferramenta de terapia para reabilitação e avaliação neuropsíquica de pacientes. Especificamente no caso de medo de altura, a situação simulada pode ser um elevador sem paredes, isto é, chão e teto invisíveis, de modo que, o paciente possa ver e se sentir dentro do elevador que, a cada sessão, sobe, e gradativamente se distancia do andar inicial proporcionando a ele a sensação de estar vivendo uma situação real. Durante as sessões, os batimentos cardíacos, pressão arterial e as reações do paciente são monitoradas pelo médico, que tem nesses dados uma importante fonte para fazer seus diagnósticos. As aplicações de terapia virtual mostram elevadas taxas de recuperação dos pacientes, onde o medo existente nas sessões iniciais, praticamente desaparece nas últimas sessões ${ }^{(14)}$.

\section{Terapia em oftalmologia: deficientes visuais}

Rodrigues, cita um simulador sem hápticos para deficientes visuais. O MoBIC Pre-Journey System (MoPS) permitia aos usuários explorar o ambiente em um mundo 
virtual simulado. A jornada simulada ajudava o usuário a se preparar para a viagem no mundo real. ${ }^{(13)}$

\section{Ensino}

Primeiro se estudaram os modelos relacionados a anatomia, deixando de lado a fisiologia e a patologia, pois essa sincronização com a fisiologia é muitas vezes negligenciada devido ao poder computacional limitado ${ }^{(15)}$. Nem todas as partes do corpo humano se beneficiam da simulação, em algumas, o estudo em cadáveres ainda é o ideal.

No ensino de anatomia usam-se muito a Realidade Virtual Passiva, onde os estudantes ou profissionais de medicina podem "passear" internamente entre os diversos órgãos do corpo humano, algo como uma endoscopia virtual, sem interferir ou modificar o ambiente. Podemos combinar imagens geradas no mundo virtual com imagens do mundo real por meio de um capacete parcialmente transparente provido de sensores. O objetivo é suplementar um cenário real com informações geradas pelo computador ${ }^{(14)}$.

A RV permite que o aprendiz desenvolva o trabalho no seu próprio ritmo, não restringe o prosseguimento de experiências ao período da aula regular, permite que haja interação, e desta forma estimula a participação ativa do estudante ${ }^{(12)}$.

Nicholson afirma que no treinamento e simulação os avanços são promissores, mas no ensino da anatomia, o uso de imagens 3D simulando a topografia do corpo humano gera controvérsias, isso porque a imagem virtual as vezes não se sobrepõe ao ensino tradicional com cadáveres ${ }^{(16)}$.

Steven ${ }^{(17)}$ já afirmava que a realidade virtual iria mudar a forma de ensinar medicina. Ela afirmava que os pacientes iriam chegar aos hospitais universitários para serem apenas cuidados e não para servir de prática dos alunos. O ensino ocorreria no simulador. Os médicos iriam calibrar os simuladores e os alunos submetidos a treinamento iriam aplicar seus conhecimentos aprendidos na vida real. Também afirmou que no futuro o uso do simulador servirá para a certificação e credenciamento. Poderia servir para averiguar os conhecimentos dos novos e velhos médicos que querem se atualizar. É claro que num futuro muito distante isso poderá ocorrer, mas algumas coisas são atuais, como o uso da simulação como certificação.

\section{Ensino: Otoscopia virtual para indicar lesões e para ensinar}

As modalidades tradicionais de ensino, tais como a dissecação de cadáveres, revelaram-se ineficazes ferramentas de ensino em algumas situações. A complexidade da orelha média e interna, juntamente com a pequena dimensão das suas estruturas anatômicas, cria muitos obstáculos para anatomia do ouvido com o ensino tradicional de ensino técnicas. Devido à pequena dimensão das amostras de ouvido e porque a orelha está dentro do osso, estudar uma orelha com a dissecação de cadáveres requer habilidades avançadas. Como alternativa, alguns educadores usam a medicina virtual ampliada ${ }^{(16)}$.

A anatomia do osso temporal é das mais complexas do organismo. O apropriado conhecimento da anatomia do osso temporal só pode ser obtido pela compreensão das inter-relações espaciais de todas as suas estruturas. Contudo, a natureza compacta do osso temporal impede a visualização direta destas estruturas. A simples descrição por palavras ou figuras em duas dimensões é de difícil compreensão. Seu conhecimento tridimensional exige muitas horas em laboratório de dissecção, tanto por parte dos estudantes quanto dos cirurgiões otológicos. A tomografia computadorizada ajudou muito nesse conhecimento anatômico, no planejamento cirúrgico, detalhando com precisão relações anatômicas que antes eram somente vistas no ato cirúrgico. A reconstrução tridimensional computadorizada tem sido utilizada por vários autores para o aperfeiçoamento do conhecimento do osso temporal ${ }^{(18)}$.

Existem cirurgias de partes do corpo que são muito perigosas (dentro do olho) ou muito pequenos (orelha interna) para ser acessíveis a instrumentos reais ${ }^{(2)}$. A principal vantagem da otoscopia virtual não é apenas para fornecer diagnósticos e informação, mas também para melhorar a qualidade e a eficiência da educação do estudante, porque contribui para uma compreensão da anatomia do ouvido médio. O uso de simuladores vem crescendo para as partes delicadas da anatomia humana, como o ouvido. Partes do corpo muito pequenas a "olho nu" são reproduzidas e aumentadas pela computação, o que permite uma visualização não conseguida com uso de peças anatômicas (os cadáveres) ${ }^{(19)}$.

\section{Ensino: Anatomia}

Nicholson afirma que existe uma tendência da diminuição do uso de cadáveres humanos para ensinar anatomia ${ }^{(16)}$. Vamos usar as aplicações da informática nos currículos da escola médica para aumentar ou, em alguns casos substituir, tradicional método de ensino, como palestras, laboratórios e livros didáticos. No domínio da anatomia humana, muitos educadores médicos voltaram-se para o ensino virtual.

Reznick explica a má aderência do tecido cadavérico que limita seu uso. $\mathrm{O}$ estudo em animais vivos também é problemática por causa das preocupações éticas, dos custos elevados, e da necessidade de instalações 
especializadas. Em contraste, modelos simuladores são seguros, reprodutíveis, portáteis, de disponível leitura, e custam geralmente menos que animais ou cadáveres ${ }^{(20)}$.

$\mathrm{Na}$ anatomia facilmente se simula uma estrutura. Quando se busca o ensino de habilidades simples, simuladores de baixa tecnologia podem ser utilizados, como por exemplo modelos anatômicos que permitam a exploração de relações entre órgãos ${ }^{(3)}$.

Currículos que não incluem cadáver nas aulas e medicina baseada em problemas estão crescendo principalmente em países de primeiro mundo, como Austrália. Foi feito um estudo em 11 escolas médicas no mundo que não usam cadáveres, 8 eram escolas de medicina na Austrália. As razões citadas para esse declínio incluem os custos crescentes e decrescentes disponibilidade de cadáveres, bem como o advento de novos métodos de ensino, tais como aprendizagem baseada em problemas, que não incluem os laboratórios de dissecção ${ }^{(16)}$.

Já o uso de simuladores para reconhecer a anatomia do corpo como método de ensino gera controvérsias. Parece que para algumas partes do corpo humanos o uso de imagens 3D melhoraria o aprendizado, como ossos do carpo, mas já para ouvido médio e interno, os resultados não foram melhores que os métodos tradicionais. Nesses estudos se aplicava um questionário após a aula expositiva. Então vemos que ainda existem controvérsias em alguns temas e algumas pesquisas contrapõem-se nos resultados, provavelmente por erro no método ou na amostra ${ }^{(16)}$.

\section{Banco de dados para anatomia: "visible humam" (VH)}

OVH é uma base de imagens bastante detalhada chamada de "Visible Humam" e composta por fotografias tiradas de fatias milimétricas obtidas através do corte de um corpo humano congelado. A partir deste conjunto de imagens de cortes bidimensionais é possível empregar algoritmos de reconstrução de imagens para extrair a anatomia $3 \mathrm{D}$ correspondente. Os modelos $3 \mathrm{D}$ obtidos a partir do $\mathrm{VH}$ passaram a servir como base para várias aplicações, pois permitem a utilização de diversos recursos gráficos como, por exemplo, passear por dentro das estruturas e aplicar efeitos como transparência para possibilitar a visualização de órgãos internos.

O projeto "Visible Human" da National Library of Medicine teve como propósito inicial criar um conjunto completo de imagens digitais tridimensionais do corpo de um adulto do sexo masculino e de um adulto do sexo feminino. Essas imagens foram obtidas em dois cadáveres, um do sexo feminino e um do sexo masculino. Esses cadáveres foram congelados e posteriormente fatiados. O cadáver masculino foi cortado em fatias de um milímetro de espessura e o cadáver feminino em fatias de 1/3 milímetro de espessura. Essas fatias foram digitalizadas e, através de técnicas de processamento de imagem, reconstruídas tridimensionalmente, formando o mais importante conjunto de imagens tridimensionais do corpo humano até hoje. Através desse conjunto de imagens, foram criadas bibliotecas e essas podem ser utilizadas por pesquisadores para a criação de ferramentas de navegação, exploração, exibição de órgãos e outras.

Para dissecar uma mulher de 59 anos (VHF), foram gastos 10 meses, tendo sido concluído em 1996, com um total de 40 GB de informação digital, resultando em um conjunto de dados mais detalhados que os correspondentes do sexo masculino de $15 \mathrm{~GB}$, completado 2 anos antes, em 1994. Isto é devido ao cadáver feminino ser cortado em intervalos de 0,33 milímetros, três vezes mais fino do que os intervalos de 1,0 milímetros sobre o cadáver do sexo masculino. Um total de 302 itens em 220 estruturas anatômicas, incluindo órgãos e partes de órgãos, vasos sanguíneos, ossos, músculos e ligamentos do abdômen e bacia do conjunto de dados VHF, foram identificados, segmentado e reconstruído ${ }^{(21)}$.

\section{Treinamento x Planejamento}

Os sistemas de planejamento permitem o estudo de um caso específico e geralmente utilizam imagens de ressonância magnética ou tomografia computadorizada do paciente para gerar uma réplica virtual da situação real. Os sistemas de treinamento objetivam a incorporação de habilidades específicas e utilizam ambientes virtuais visando preparar o usuário para realizar um determinado procedimento, podendo simular situações genéricas (desassociadas das peculiaridades de um paciente específico) com alto grau de realismo.

\section{Pacientes Virtuais}

Os diagnósticos por imagem na área médica já se estabeleceram há algum tempo com uma variedade de equipamentos para aquisição de imagens como tomografia computadorizada, ressonância magnética e ultrassonografia. Muitas vezes, a simples análise visual da imagem não é suficiente para determinar as características das estruturas em estudo e torna-se necessário obter modelos geométricos que representem essas estruturas e que possibilitem a extração de medidas e a simulação de procedimentos. Com base nessa necessidade, uma linha seguida por diferentes grupos é a construção dos chamados pacientes virtuais que são modelos de representação de humanos virtuais, ainda que apenas parciais, para uso de aplicações de Compu- 
tação Gráfica e RV na área médica. Pesquisas voltadas a esta área visam a permitir o melhor entendimento da forma humana bem como suas funções e desenvolvimento. Através desses sistemas, um estudante poderia participar repetidas vezes de situações raras ou de emergência ${ }^{(22)}$.

Divide-se a construção de "humanos virtuais" em três etapas básicas:

- obtenção das imagens médicas;

- reconstrução tridimensional do modelo;

- simulação de movimento e deformações.

A primeira etapa consiste na aquisição e processamento das imagens médicas para a reconstrução tridimensional das partes que compõem o corpo humano. Nesta etapa são utilizadas técnicas para melhoria das imagens e correção de registro. Na segunda etapa, métodos de modelagem geométrica, segmentação e topologia de elementos básicos como ossos, pele, músculos, tendões, vasos, gordura, etc., fornecem os componentes fundamentais para a construção do corpo 3D. Finalmente, a última etapa é a simulação do movimento e deformação dos tecidos. Para esta etapa são necessários algoritmos que simulam $\operatorname{corpos}^{(22)}$.

\section{Treinamento em oftalmologia: cirurgia de catarata}

Shen criou um simulador híbrido que permite treinar a cirurgia da catarata. A simulação consiste em inserir outra ferramenta especializada dentro da córnea para quebrar a lente envelhecida e desenhar as suas sobras. Finalmente, a lente artificial é inserida no lugar da antiga ${ }^{(23)}$.

\section{Exames: Otoscopia virtual}

Em humanos, o diagnóstico da doença da orelha foi alcançado com endoscopia virtual, bem como a radiografia, a tomografia computadorizada, ou RM. A endoscopia virtual permite inspeção visual da finas estruturas do osso temporal e não necessita de mais dados do que a tomografia computadorizada de rotina, mas fornece informação topográfica 3D virtual endoscópica. Tem sido usada para diagnóstico, planejamento cirúrgico, e o ensino nas escolas ${ }^{(19)}$.

\section{Oftalmologia e o futuro da medicina virtual}

$\mathrm{Na}$ oftalmologia se tem buscado mais do que o uso de atlas tridimensionais e não simuladores. Isso se deve a dificuldade de se criar um simulador com semelhança ao olho humano, e continua sendo uma área, como no setor da otoscopia, em que a simulação baseada em ferramentas mecânicas, com hápticos, não melhora o desempenho dos alunos. Como o olho humano é muito delicado, o simulador deverá ser muito delicado, e isto dificulta a criação de um material deste porte.

Em algumas áreas, o uso de simuladores para treinamento não foi comprovado o benefício em relação aos métodos de ensino clássico.

Apesar de amplamente utilizado por algum tempo em outros campos, como a formação aeronáutica, a tecnologia de RV tem sido recentemente proposta como uma poderosa ferramenta para a educação de saúde profissionais $^{(24)}$.

Atualmente, tanto o conhecimento médico quanto o computacional disponível é limitado. Este fato devese em parte à complexidade da medicina e, em outra parte, às tecnologias de computação em estado constante de aperfeiçoamento e expansão. O dramático progresso na disseminação de informação e nas tecnologias de comunicação através da Internet e das redes de computadores afetará consideravelmente o ensino médico nos próximos anos ${ }^{(25)}$.

Soferman ${ }^{(7)}$ já afirmava que com gráficos, memórias e texturas com maior largura de banda se tornarão disponíveis, o desempenho do sistema irá aumentar ao ponto em que os dados de imagem representando a totalidade do corpo humano poderão ser armazenados na resolução máxima.

Os desafios para o futuro são: interação homem máquina em tempo real, qualidade digital de imagem, a transmissão de dados maciços, armazenamento e interfaces para interagir com os dados.

\section{REFERÊNCIAS}

1. Machado LS. Conceitos básicos da realidade virtual. São José dos Campos: Instituto Nacional de Pesquisas Espaciais; 1995.

2. Satava RM, Jones SB. Current and future applications of virtual reality for medicine. Proc IEEE. 1998;86(3):484-489.

3. Pazin Filho A, Scarpelini S. Simulação: definição. Medicina (Ribeirão Preto). 2007;40 (2):162-6.

4. Hammoud MM, Nuthalapaty F, Goepfert AR, Casey PM, Emmons S, Espey EL. Kaczmarczyk JM, Katz NT, Neutens JJ, Peskin EG; Association of Professors of Gynecology and Obstetrics Undergraduate Medical Education Committee. To the point: medical education review of the role of simulators in surgical training. Am J Obstet Gynecol. 2008;199(4):338-43.

5. Suzuki S, Suzuki N, Hattori A, Hayashibe M, Konishi K, Kakeji Y, Hashizume M. Tele-surgery simulation with a patient organ model for robotic surgery training. Int $\mathbf{J}$ Med Robot. 2005;1(4):80-8.

6. Dawson SL, Kaufman JA. The imperative for medical simulation. Proc IEEE. 1998;86(3):479-83.

7. Soferman Z, Blythe D, John NW. Advanced graphics behind medical virtual reality: evolution of algorithms, hardware, and software interfaces. Proc IEEE. 1998;86(3):531-54.

8. Letterie GS. How virtual reality may enhance training in obstetrics and gynecology. Am J Obstet Gynecol. 2002;187(3 Suppl):S37-40. Review. 
9. Nunes FLS, Machado LS, Pinho MS, Kirner C, organizadores. Abordagens práticas de realidade virtual e aumentada. In: XI Symposium on Virtual and Augmented Reality.Porto Alegre: Sociedade Brasileira de Computação; 2009. p. 31-68.

10. Savi R, Ulbricht VR. Jogos digitais educacionais: benefícios e desafios. Novas Tecnol Educ. 2008;6(2).

11. Rosen JM, Soltanian H, Redett RJ, Laub DR. Evolution of virtual reality. IEEE Eng Med Biol Mag. 1996;15(2):16-22.

12. Braga M. Realidade virtual e educação. Rev Biol Ciênc Terra. 2001;1(1).

13. Rodrigues CEM. Um dispositivo háptico de auxílio à navegação para deficientes visuais [trabalho de conclusão de curso]. Recife: Centro de Informática da Universidade Federal de Pernambuco; 2006.

14. Mergulhão LC, Mokonuma SL, Silva Cruz TA. Ambiente virtual em situações críticas: análise de estudo de caso com base na estrutura e modelagem de sistemas de realidade virtual [trabalho de conclusão de curso]. Belém: Curso de Bacharelado em Ciência da Computação. Centro de Ciências Exatas e Tecnologia da Universidade da Amazônia; 2003.

15. Dumay ACM. Beyond medicine. IEEE Eng Med Biol. 1996;15(2):34-40.

16. Nicholson DT, Chalk C, Funnell WR, Daniel SJ. Can virtual reality improve anatomy education? A randomised controlled study of a computer-generated three-dimensional anatomical ear model. Med Educ. 2006;40(11):1081-7.

17. Dawson SL, Kaufman JA. The imperative for medical simulation. Proc IEEE. 1998; 86( 3):479-83.
18. Bogar P, Amaro Júnior E, Bento RF, Miniti A. Reconstrução tridimensional de acessos cirúrgicos para neurimoma do acústico, utilizando imagens de tomografia computadorizada. Rev Bras Otorrinolaringol. 1998;64(1):20-4.

19. Eom K, Kwak H, Kang H, Park S, Lee H, Kang H, et al. Virtual CT otoscopy of of the middle ear and ossicles in dogs. Vet Radiol Ultrasound. 2008;49(6):545-50.

20. Reznick RK, MacRae H. Teaching Surgical Skills - Changes in the Wind. N Engl J Med. 2006;355:2664-9.

21. Bajka M, Manestar M, Hug J, Székely G, Haller U, Groscurth P. Detailed anatomy of the abdomen and pelvis of the visible human female. Clin Anat. 2004;17(3):252-60. Erratum in: Clin Anat. 2004;17(5):450.

22. Ramos FM. Aplicação de realidade virtual para construção de atlas de anatomia e fisiopatologia do câncer de mama [dissertação]. Marília: Centro Universitário Eurípides de Marília; 2005. 98 f.

23. Shen X, Zhou J, Hamam A, Nourian S, El-Far NR, Malric F, Georganas ND. Haptic-enabled telementoring surgery simulation. IEEE MultiMedia. 2008;15(1):64-76.

24. Suebnukarn S, Phatthanasathiankul N, Sombatweroje S, Rhienmora P, Haddawy P. Process and outcome measures of expert/novice performance on a haptic virtual reality system. J Dent. 2009;37(9):658-65.

25. Rodrigues MA. Aplicações de novas tecnologias de computação para simulação e treinamento de procedimentos cirúrgicos. In: XXIX Seminário Integrado de Software e Hardware (SEMISH). Florianópolis: Sociedade Brasileira de Computação; 2002 